\title{
Spontaneous Septic Monoarthritis of the Scaphoid- trapezium Joint Presenting as Septicaemia and Death in an Elderly Female: A Case Report
}

\author{
VK Singh, D Orth MRCS, PK Singh*, MS, MRCS \\ Department of Trauma \& Orthopaedics Surgery, James Paget University Hospital, United Kingdom \\ * Royal Hallamshire Hospital, United Kingdom
}

\begin{abstract}
Primary involvement of carpal bones in septic arthritis is a rare event. We report here a case of idiopathic septic arthritis with isolated involvement of the scaphoid-trapezium joint. An elderly patient presented with collapse and symptoms of septicaemia, and right wrist that was swollen and tender. Radiographs revealed erosion of the scaphoid-trapezium joint and increased joint space. The joint was aspirated yielding frank pus. Swelling and redness improved, but the patient died due to ongoing septicaemia. Localised septic arthritis of the scaphoid-trapezium joint, however rare, can be fatal if not aggressively treated in a timely fashion.
\end{abstract}

Key Words:

Septic mono-arthritis; Scaphoid-trapezium joint

\section{INTRODUCTION}

Less than $10 \%$ of septic arthritis cases affect the wrist and those involving the carpal bones are even more rare. Penetrating injury is the most frequently seen cause of septic arthritis, followed closely by haematogenous spread of infection ${ }^{1,2}$. Seeding of the synovium usually occurs in the presence of one or more risk factors that lead to immunosuppression ${ }^{2}$. Spontaneous, idiopathic septic arthritis of carpal joints is a rare entity; in fact a search of the literature yielded only three reported cases ${ }^{1,2,3}$. Isolated involvement of the scaphoid-trapezium joint in septic arthritis without any predisposing factor has not yet been described.

\section{CASE HISTORY}

An otherwise healthy 82-year-old female was admitted to emergency department due to collapse. The only complaint was a painful swollen right wrist for the week prior to admission. There was no history of recent trauma or other medical conditions. On admission, the patient was febrile, with white blood cell count of $20,000 / \mathrm{mm}^{3}$ (neutrophils $17,500 / \mathrm{mm}^{3}$ ), erythrocyte sedimentation rate at $42 \mathrm{~mm} / \mathrm{hr}$ and a high C-reactive protein of 299 units. Blood culture was sent along with a complete septic screen. Urine dipstick and echocardiogram were unremarkable. With septicaemia being the first impression, the patient was initially managed with administration of broad-spectrum intravenous antibiotics.

The patient was then referred to the orthopaedic team for further management. On examination the wrist was swollen, red and warm with maximum tenderness localised at the scaphoid-trapezium joint. Radiographs revealed erosion of scaphoid-trapezium joint with increased joint space (Fig. 1). A provisional diagnosis of septic intercarpal arthritis was made. The scaphoid-trapezium joint was aspirated under general anaesthesia using fluoroscopic guidance (Fig. 2). Frank pus was removed resulting in decreased joint space (Fig. 3). The joint was thoroughly irrigated with saline, and aspirate was sent for microbiological examination; the wrist was then immobilised in a back slab. Intravenous antibiotics were continued pending a definitive culture report. The cultures were negative but Gram staining demonstrated the presence of Gram-positive cocci. With the advice of a microbiologist, intravenous antibiotics were started empirically to treat Staphylococcus aureus. Although local swelling and redness reduced substantially with the new antimicrobial therapy, systemic symptoms barely improved. Unfortunately, the patient died of continuing septicaemia two days of the new treatment regimen.

\section{DISCUSSION}

The relative rarity of septic arthritis of the carpal joints is a poorly understood but widely agreed upon ${ }^{1,2,4}$. Isolated involvement of the scaphoid-trapezium joint has, to our knowledge, never been reported. Approximately $75 \%$ of nongonococcal septic arthritis is due to gram-positive cocci, with Staphylococcus aureus the most common organism ${ }^{1,4}$.

Unlike the case reported herein, most previously reported cases had a demonstrable portal of entry ${ }^{1,2}$. Penetrating trauma is the most common identified cause, with few reports of spontaneous involvement without any

Corresponding Author: VK Singh, Registrar Trauma and Orthopaedics, Luton and Dunstable Hospital NHS Trust Lewsey Road, Luton, LU4 ODH, United Kingdom Email: we.publish@googlemail.com 


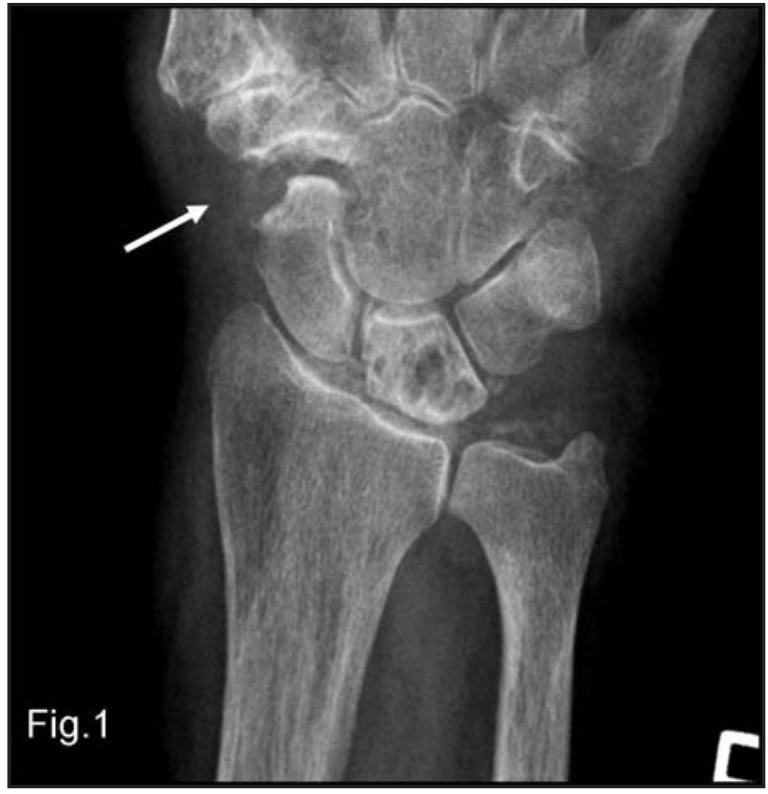

Fig. 1: Preoperative X-Ray of the wrist, revealing widening and erosion of the scaphoid-trapezium joint.

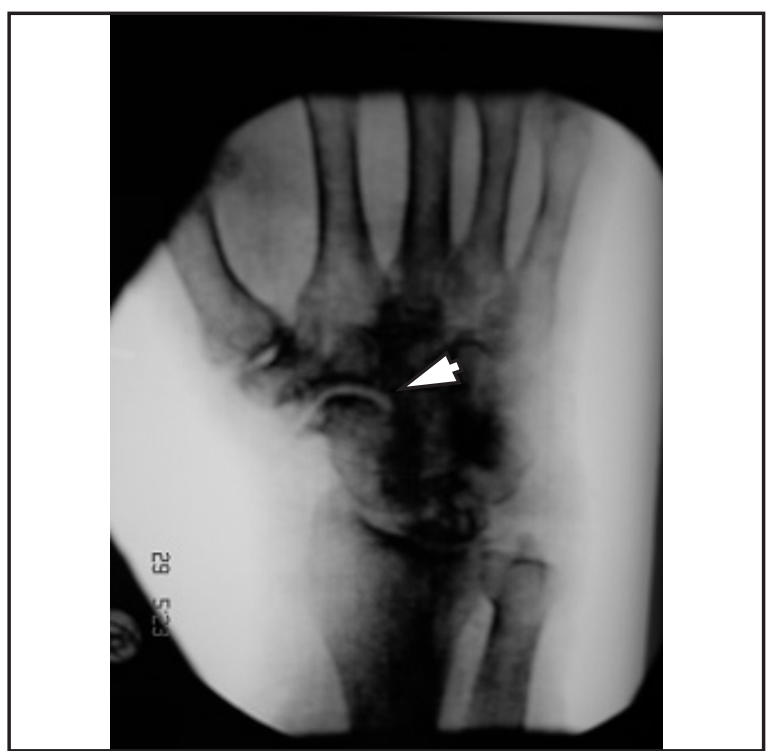

Fig. 3: Resultant decrease in joint space immediately after aspiration (arrow head).

predisposing factors ${ }^{1,2}$. Haematogenous spread to the joint from an unknown focus could be a possible explanation in the current case. Staphylococcus aureus is found as a commensal organism of human nares in $25 \%$ of the population $^{5}$, thus raising the possibility that in this case, the patient's infection came from a human carrier of the organism.

A high index of suspicion and aggressive treatment are required for early diagnosis and correct treatment. In fact,

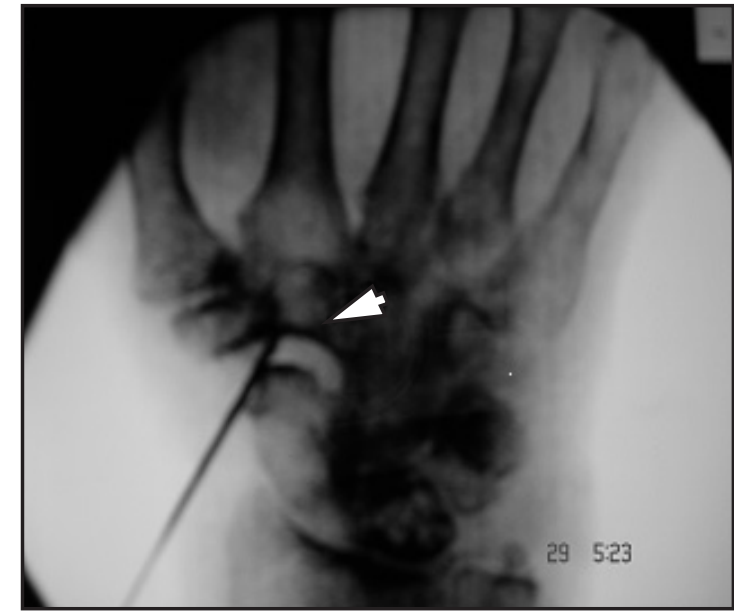

Fig. 2: Intraoperative fluoroscopic image of right wrist revealing isolated involvement of the scaphoid-trapezium joint with increased joint space (arrow head). The aspiration needle is visible in situ.

Rashkoff et al. advocate early dorsal aspiration using an 18gauge needle ${ }^{1}$. Culture and Gram staining remain the standard techniques for diagnostic confirmation ${ }^{1}$.

Parenteral antibiotics are invaluable tools in managing cases such as the one reported here, but it is important to send the aspirate for gram staining and culture before embarking upon such a course of action. On the other hand, early intravenous antibiotics may mask symptoms leading to false negative cultures and delay in appropriate treatment ${ }^{4}$. Physicians generally consider aspiration to be a diagnostic and not a curative procedure, but in cases such as this, early surgery should be considered. Creation of a window over the extensor surface of the involved joint provides a portal for drainage and allows enough stability to initiate early mobilisation ${ }^{1}$. With definitive correlation between the timing of surgery and final outcome in this case, we consider septic arthritis of carpal joints to be a surgical emergency requiring immediate arthrotomy. All patients with good outcome in the Rashkoff et al. study underwent surgery within 10 hours of diagnosis ${ }^{1}$.

As aspiration has a diagnostic as well as therapeutic value, the authors advocate early aspiration of the wrist followed by urgent arthrotomy and early postoperative rehabilitation in cases such as the one presented here. Septic arthritis of the scaphoid-trapezium joint should be the differential diagnosis when dealing with infective conditions in the region of the wrist joint. Furthermore, this case shows that septic arthritis of even a small joint such the scaphoid-trapezium, cannot be underestimated and can be fatal if not diagnosed and treated promptly. 


\section{REFERENCES}

1. Rashkoff ES, Burkhalter WE, Mann RJ. Septic arthritis of the wrist. J Bone Joint Surg (Am). 1983; 65: 824-8.

2. Chin KR, Spak JI, Jupiter JB. Septic arthritis and osteomyelitis of the wrist: reconstruction with a vascularized fibular graft. $J$ Hand Surg (Am). 1999; 24(2): 243-8.

3. Gerardi JA, Mack GR, Lutz RB. Acute carpal tunnel syndrome secondary to septic arthritis of the wrist. J Am Osteopath Assoc. 1989; 89: 933-4.

4. Gelman MI, Ward JR. Septic arthritis: a complication of rheumatoid arthritis. Radiology. 1977; 122: 17-23.

5. Benenson AS, ed. Control of communicable diseases manual. Washington, DC: American Public Health Association, 1995; 42932 . 